\title{
Matrices de microgouttes pour l'analyse de globules rouges uniques
}

\author{
Paul ABBYAD et Antigoni ALEXANDROU \\ Laboratoire d'Optique et Biosciences - École polytechnique, CNRS, INSERM U696 \\ paul.abbyad@polytechnique.edu•antigoni.alexandrou@polytechnique.edu \\ Rémi DANGLA et Charles BAROUD \\ Laboratoire d'Hydrodynamique - École polytechnique, CNRS \\ remi.dangla@polytechnique.org•baroud@ladhyx.polytechnique.fr
}

La micro-fluidique a le potentiel pour révolutionner la pratique des sciences de la santé à travers la miniaturisation et l'intégration des briques élémentaires des laboratoires de biologie. Ces nouvelles technologies, en cours de développement, permettront d'intégrer sur une puce unique des opérations qui nécessitent actuellement des robots, des instruments de mesure coûteux, en plus des heures de travail pour le personnel de laboratoire. On parle donc de Laboratoires sur puce, ou «Lab on a chip» en anglais, qui visent à automatiser et à standardiser les opérations du laboratoire de biologie.

\section{Manipuler des microgouttes}

La manipulation de petits volumes de fluides biologiques constitue une brique de base qui doit être maitrisée comme prérequis à toute analyse. Une solution émergente est la manipulation avancée de gouttes ayant un volume de quelques nano-litres chacune. Ces gouttes peuvent contenir les échantillons ou des cellules à analyser; elles sont produites dans une huile inerte qui sert aussi à les transporter dans un réseau de microcanaux. De cette façon, chaque goutte peut servir de microréacteur dans lequel peut se dérouler une réaction chimique ou biologique. Les réactions sont ainsi naturellement isolées de leurs voisines par l'huile porteuse et le contenu de la goutte est préservé au cours du temps

\section{L'étude de la drépanocytose}

De plus, les systèmes micro-fluidiques représentent une plateforme idéale pour l'observation et la manipulation des cellules du système sanguin car ils permettent de s'approcher des conditions physiologiques en contrôlant le flux, la concentration d'oxygène, les forces de cisaillement et la géométrie des canaux. Nous avons choisi d'aborder la dynamique des globules rouges dans le cas d'une maladie génétique qui touche en majorité les populations d'origine Africaine : la drépanocyłose ou anémie falciforme (encadré 1). Pour cela, nous avons réalisé une nouvelle plateforme pour l'étude de la maladie à l'échelle de la cellule individuelle. Cette approche permet l'observation et l'étude d'un grand nombre de globules rouges individuels alors qu'ils sont soumis à des cycles d'oxygénation/désoxygénation, en présence ou non de molécules-médicaments candidates.

La drépanocyłose est caractérisée par un changement de forme drastique du globule rouge, la falciformation, lorsque celui-ci se trouve dans des conditions d'hypoxie. Ces cellules falciformes sont rigides et peuvent entraîner un blocage des vaisseaux capillaires. Les globules rouges subissent des cycles d'oxygénation/désoxygénation durant leur circulation entre les poumons et les tissus. Notre but a été de reproduire ces cycles d'oxygène physiologiques, tout en observant les globules rouges individuellement, afin d'étudier la falciformation de globules rouges et les effets cumulatifs dus à la falciformation répétitive.

\section{Le dispositif microfluidique}

L'approche développée est présentée schématiquement (figure la) et en photo (figure 1b). Le flux de fluides, l'huile et la solution aqueuse, sont contrôlés en débit ov en pression. La pression partielle d'oxygène des fluides est fixée avant l'entrée dans le dispositif par un échange de gaz dans des flacons à atmosphère contrôlée. Les fluides sont ensuite introduits dans le dispositif microfluidique. En poussant I'huile et la solution aqueuse par un rétrécissement, des gouttes de tailles identiques (dans la gamme 100 à $300 \mu$ m, soit de 1 à $10 \mathrm{~nL}$ ) sont produites à un rythme constant (figure 1c). Ces gouttes entrent dans un large canal et sont piégées en matrice (figure 1d) dans des positions prédéfinies, en exploitant un gradient d'énergie de surface qui les maintient stationnaires face à un écoulement d'huile (encadré 2).

\section{Reproduire des cycles d'oxygène}

Cette approche micro-fluidique permet de réaliser un contrôle précis et efficace du taux d'oxygène à l'intérieur des gouttes, en exploitant l'échange de gaz entre 


\section{La drépanocyłose}

La drépanocytose (ou anémie falciforme) est la maladie génétique la plus courante en France avec environ 10000 malades et quelques millions dans le monde. En Afrique, selon les pays, il y a de 10 à $40 \%$ de porteurs sains. Dans le monde, on estime qu'il y a de 200000 à 300000 nouveau-nés par an qui souffrent de la maladie drépanocytaire, nombre résultant d'une projection car la plupart vont mourir avant 5 ans sans diagnostic ou de malnutrition sans que le diagnostic réel de maladie sous-jacente soit porté. En France, il faut rappeler que l'on a dépassé les 300 naissances par an depuis 2003 avec une majorité de naissances en Ile-de-France, aux Antilles et en région PACA. Aux ÉtatsUnis, on considère qu'il y a une naissance d'enfant atteint sur 600 naissances dans la communauté Afro-américaine.

Depuis les travaux de Linus Pauling en 1949 et de Vernon Ingram en 1956 on sait qu'une mutation génétique de l'hémoglobine est responsable de cette maladie. Elle affecte l'hémoglobine, la molécule qui sert à transporter l'oxygène dans nos globules rouges (figure a) et qui leur donne leur couleur. Durant la circulation san- guine, I'hémoglobine lie l'oxygène à son passage dans les poumons, pour ensuite le relarguer dans les tissus. Dans le cas d'une personne atteinte de la drépanocytose, 'hémoglobine dé-oxygénée présente une zone hydrophobe à sa surface qui provoque sa polymérisation, entraînant ainsi un changement de forme et des propriétés mécaniques du globule rouge. Les globules rouges peuvent prendre une forme de faucille (figure b), d'où le nom de la maladie (drepanos = faucille en grec), deviennent plus rigides et moins déformables et peuvent ainsi se bloquer dans les petits vaisseaux capillaires. Le blocage des globules rouges rigides dans les capillaires provoque des infarctus ou des «crises vaso-occlusives» (figure $\mathrm{c}$ ), très douloureuses, qui lèsent les organes et contribuent à la plus faible espérance de vie des patients.

Àl'heure actuelle, le seul traitement curatif est la greffe de moelle osseuse provenant d'un donneur compatible. Malheureusement, peu de patients peuvent bénéficier de ce traitement. Alternativement, des stratégies médicamenteuses pouvant bénéficier au plus grand nombre sont encore en phase de recherche. C'est le cas de stratégies thérapeutiques visant à retarder le processus de polymérisation del'hémoglobine au sein des globules rouges ou d'empêcher la déshydratation de cellules et le dommage des membranes cellulaires dus à la falciformation répétitive.

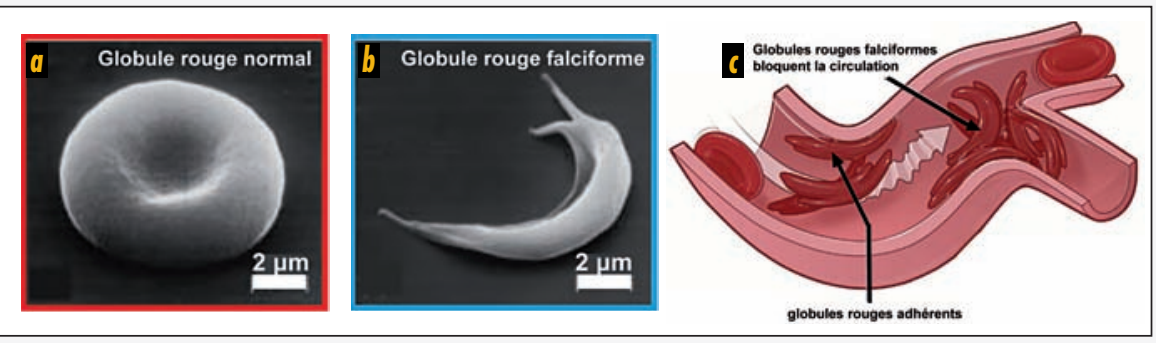

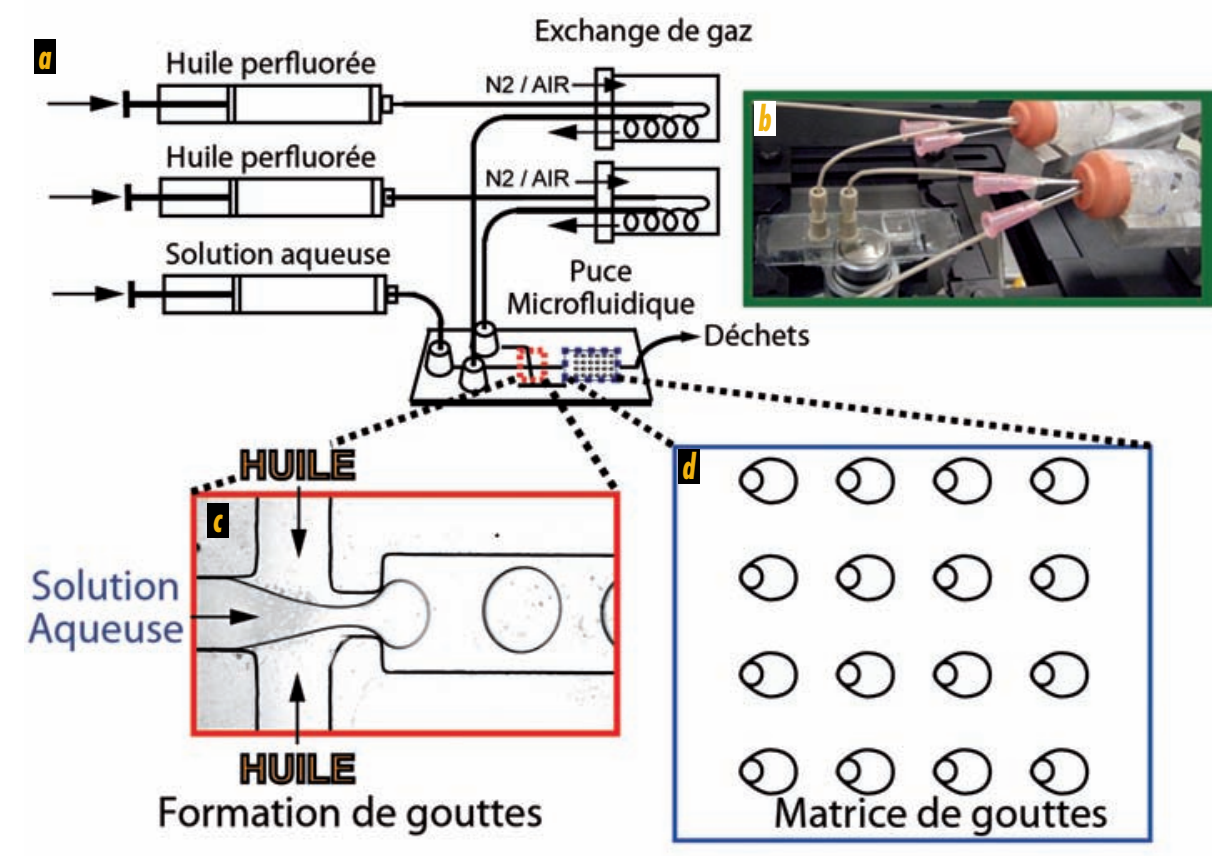

Figure 1. a. Schéma de l'expérience : contrôleur de débit, flacons pour ajuster le taux d'oxygène et le dispositif micro-fluidique. b. Photo des flacons d'échange de gaz et de la puce micro-fluidique placée sur le microscope. c. Formation de gouttes aqueuses lors de l'écoulement de l'huile et de la solution aqueuse par un rétrécissement du canal micro-fluidique. do Les gouttes sont piégées dans une matrice bidimensionnelle. les gouttes piégées et l'huile en écoulement. En variant les débits relatifs de deux entrées d'huile avec des pressions partielles d'oxygène différentes (tout en gardantle débit total fixe), nous pouvons alors soumettre les gouttes à des cycles de pression partielle d'oxygène (figure 2a). Cette variation du niveau d'oxygénation peut être vérifiée à travers des mesures en fluorescence, en diluant une sonde fluorescente dans les gouttes. La durée de vie de fluorescence de cette sonde est corrélée à la pression partielle d'oxygène et permet de suivre la progression du taux d'oxygène d'une goutte unique (figure 2b).

\section{La falciformation des globules rouges dans les microgouttes}

Les globules rouges provenant de patients atteints de la drépanocytose sont placés dans des gouttes micro-fluidiques, avec un petit nombre de cellules par goutte. La polarisation de la lumière et 


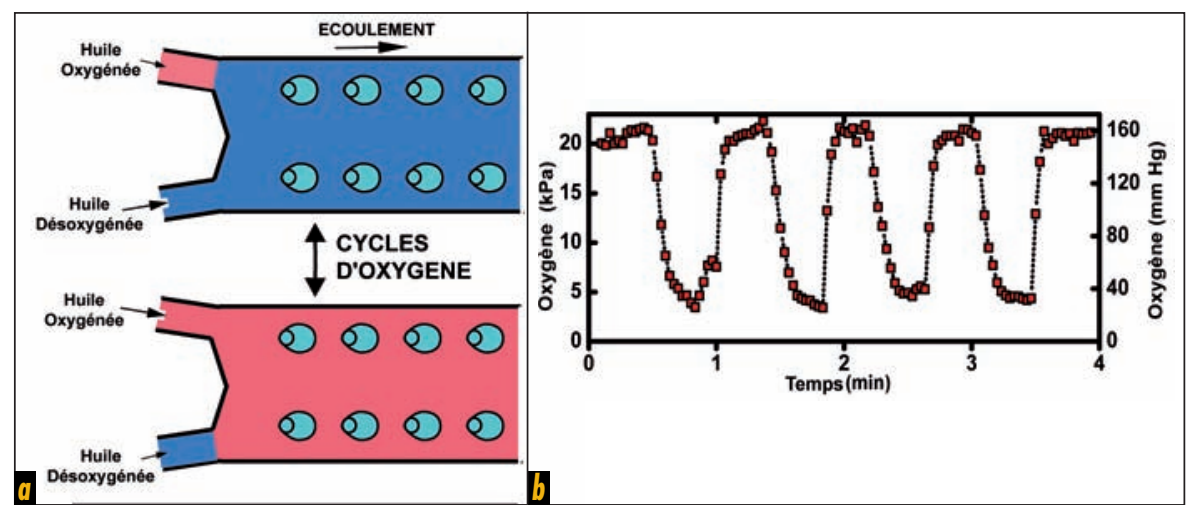

\section{Encadré 2 Le piégeage de microgouttes}

Le piégeage de gouttes s'appuie sur une réduction de l'énergie de surface. Les gouttes ne sont pas sphériques, mais plutôt en forme de disques, confinés par les surfaces supérieure et inférieure du canal. Des puits sont microfabriqués au fond $d u$ canal. La goutte s'étend dans ce puits,

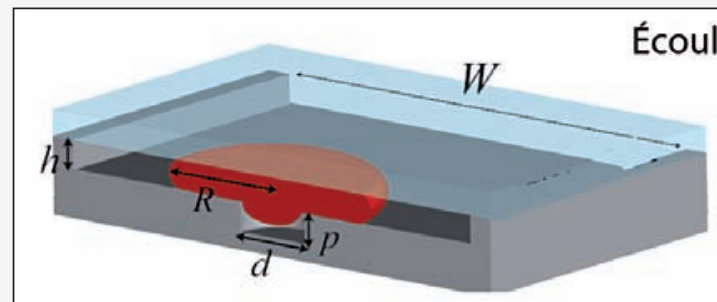

a

menantà une réduction de l'énergie de surface de la goutte (figure a). La goutte est alors immobilisée malgré la force d'entrainement de l'huile (figure b). L'utilisation de ces micro-géométries novatrices permet d'observer le contenu des gouttes en continu durant plusieurs heures.

\section{Écoulement}

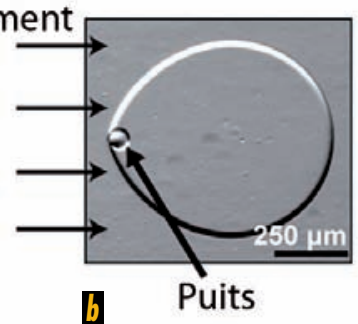

\section{Encadré 3 Détection de falciformation par la biréfringence}

La détection de la falciformation par biréfringence est rendue possible par les propriétés optiques de l'hème, molécule plane responsable de la fixation de l'oxygène à l'intérieur de l'hémoglobine. L'hème absorbe préférentiellement la lumière polarisée parallèlement à son plan. Des molécules $d$ 'hémoglobine en solution ne présentent pas de biréfringence puisque les différentes molécules $d$ 'hème sont orientées aléatoirement. Lorsque des polymères d'hémoglobine $S$ se forment dans des globules rouges drépanocytaires (figure a), leur rotation à l'intérieur du globule rouge n'est plus libre et une biréfringence apparaît. Ainsi, des globules rouges normaux observés en transmission entre deux polariseurs croisés apparaissent noirs alors que des globules rouges drépanocytaires apparaissent clairs (figure b). Ces observations sont possibles parce que la vitesse $d^{\prime}$ acquisition des images est plus rapide que la rotation des globules rouges. Cette technique permet de distinguer aisément les cellules comportant des polymères d'hémoglobine.

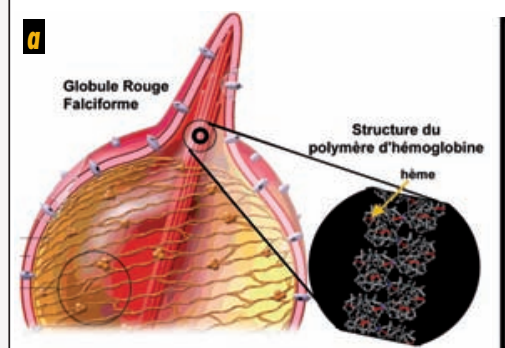

Adaptée de Frenette et al., J Clin Invest., 2007

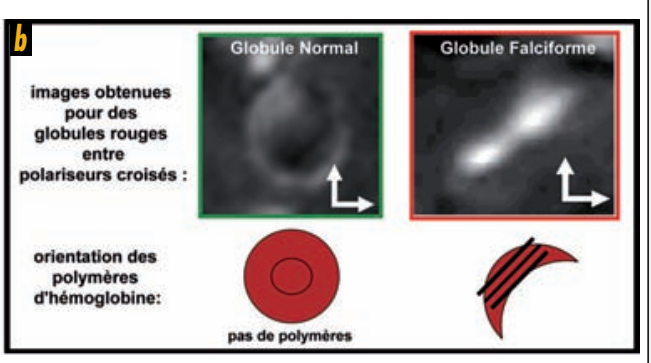

Adaptée de Harrington et al., J. of Molecular Biology 1997
Figure 2. a. Des cycles de la pression partielle $d^{\prime}$ 'oxygène des gouttes sont obtenus en modulant l'écoulement d'huile oxygénée et désoxygénée. b. Des cycles de pression partielle d'oxygène d'une goutte unique induits par l'échange avec I'huile en écoulement. Mesures obtenues à l'aide $d^{\prime}$ un fluorophore à base de ruthénium (RDTP) dont la durée de vie de l'émission dépend de la concentration partielle d'oxygène.

les propriétés de biréfringence de l'hémoglobine sont exploitées pour détecter la polymérisation de l'hémoglobine à l'intérieur des globules rouges drépanocytaires en fonction des conditions de l'expérience (encadré 3). Cette méthode permet de s'affranchir des difficultés associées à la détection de la falciformation par la morphologie des globules rouges qui s'avère difficile lorsque ceux-ci sont en écoulement. Ainsi, la détection de polymères d'hémoglobine dans les globules rouges falciformes se fait par simple mesure d'intensité lumineuse entre polariseurs croisés.

L'oxygénation des cellules est alors contrôlée en modulant le contenu en oxygène de l'huile qui entoure les gouttes. Grâce à l'écoulement simultané de deux huiles avec des pressions partielles d'oxygène différentes, on peut obtenir une désoxygénation sélective d'une rangée de gouttes (figure $3 a$ ). On distingue alors des globules falciformes en blanc dans la goutte désoxygénée, tandis que les globules oxygénés apparaissent noirs sur l'image. Le contrôle sélectif du taux d'oxygène d'une matrice de gouttes permet la comparaison, en temps réel, des globules rouges dans différentes conditions d'oxygène.

En modifiant les débits des deux entrées d'huile, on produit des cycles d'oxygénation et de désoxygénation dans les gouttes. On observe alors des transitions de polymérisation/dépolymérisation en fonction du taux d'oxygène (figure 3b). Ceci nous permet de déterminer le décalage entre la désoxygénation et la falciformation des globules rouges, un paramètre critique puisqu'il détermine si les globules rouges falciforment dans la microcirculation ou retournent au poumon sans incident. 

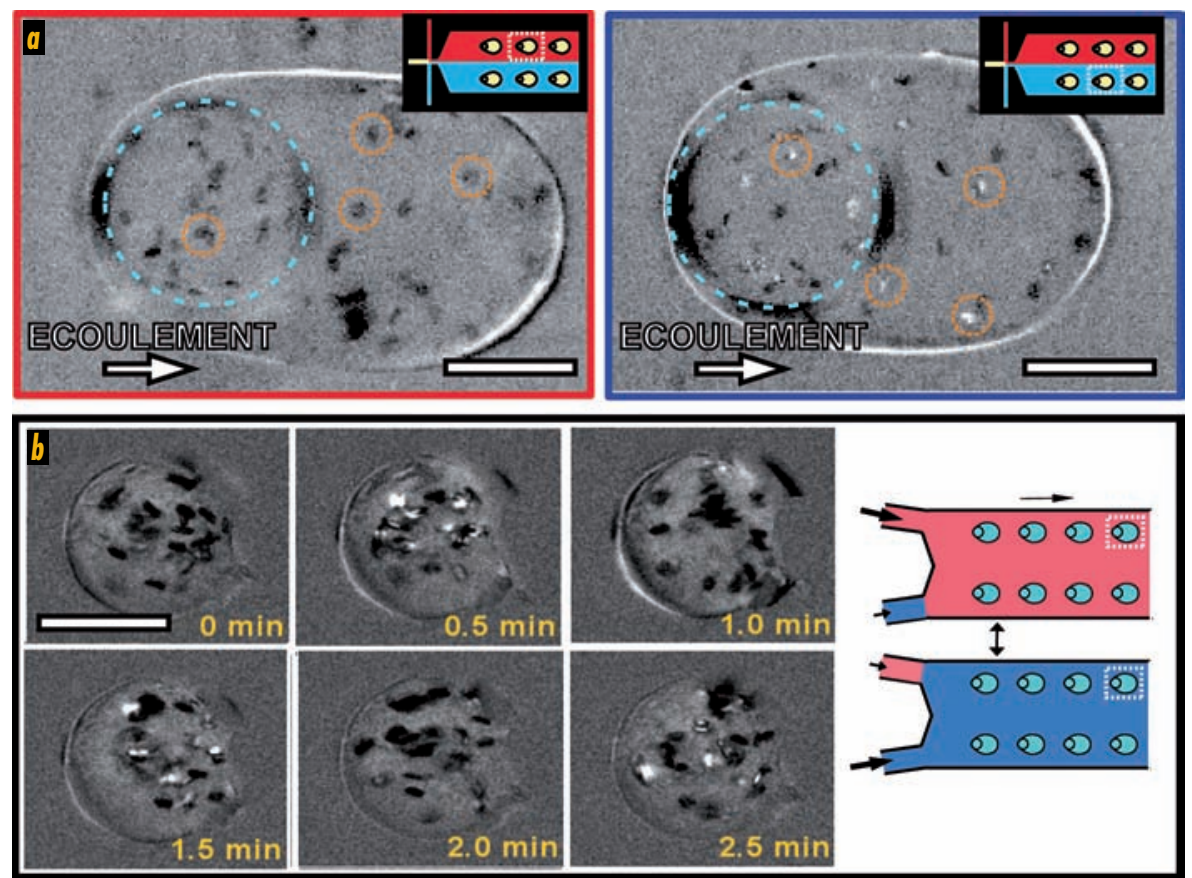

Figure 3. Images de gouttes contenant des globules rouges placées entre polariseurs croisés. a. Un flux laminaire $\mathrm{d}^{\prime}$ un système micro-fluidique permet la désoxygénation sélective d'une rangée de gouttes. Une goutte oxygénée est présentée à gauche et une goutte désoxygénée à droite. Le schéma en encartindique la position relative de la goutte dans la matrice. Le trait bleu indique l'emplacement du piège. Quelques globules rouges sont encerclés en orange. Les globules rouges contenant des polymères d'hémoglobine apparaissent blancs alors que les globules rouges sans polymères apparaissent noirs. b. Gouttes microfluidiques contenant des globules rouges, soumises à des conditions d'oxygénation ou désoxygénation contrôlées. Cela provoque la polymérisation et dépolymérisation de l'hémoglobine à l'intérieur des globules rouges mise en évidence par les transitions de noir en blanc en fonction du taux d'oxygène de la goutte. Le schéma de droite explique comment sont produits les cycles d'oxygénation et désoxygénation des gouttes (voir aussi figure 2A). Barre d'échelle dans les images : $100 \mu \mathrm{m}$.

\section{Perspectives}

Cette nouvelle plateforme permettra d'étudier de façon quantitative la falciformation de globules rouges uniques en fonction du cycle de désoxygénation et en présence de molécules-médicaments candidates. II s'agit, en particulier, d'explorer les modifications de la membrane cellulaire, le rôle de la déshydratation induite par la déformation radicale du globule rouge et l'importance des interactions intercellulaires dans le processus de vaso-occlusion.

Grâce au contrôle précis des paramètres physiques et biochimiques qu'elles offrent, les puces microfluidiques présentées ici permettent l'observation de la réponse de cellules uniques à des stimulations extérieures. Dans le domaine de la santé plus généralement, les systèmes microfluidiques sont utilisés aujourd'hui pour développer des approches intégrées. L'on tente de s'approcher des vaisseaux sanguins en incorporant une couche de cellules endothéliales voire de reproduire des organes tels que la rate ou le poumon. L'ambition de ces systèmes est de comprendre le fonctionnement et les causes de dysfonctionnement de ces organes et ainsi apporter de nouvelles pistes pour le traitement de diver ses pathologies.

\section{Références}

[1] P. Abbyad, P.-L. Tharaux, J.-L. Martin, C.N. Baroud, A. Alexandrou, Sickling of Red Blood Cells through Rapid Oxygen Exchange in Microfluidic Drops, Lab Chip 10, 2481-2636 (2010).

[2] P. Abbyad, R. Dangla, A. Alexandrou, C.N. Baroud, Rails and Anchors: Guiding and Trapping Droplet Microreactors in Two Dimensions, Lab Chip 11, 813-821 (2011).

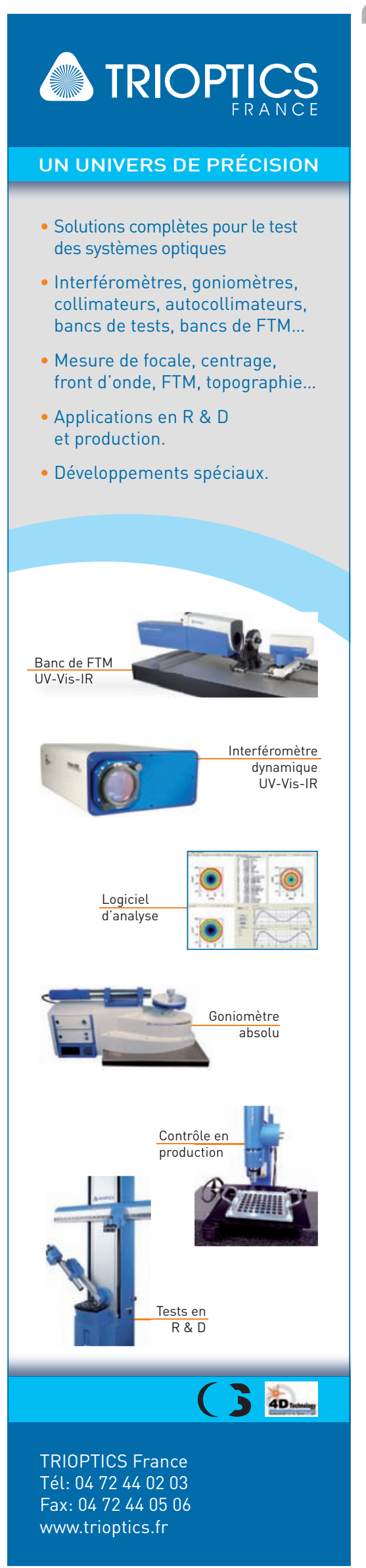

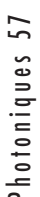

